\title{
Die Last der Geschichte
}

\begin{abstract}
Was können geschichtswissenschaftliche Forschungen zur Aufarbeitung des sexuellen Missbrauchs an Minderjährigen in der katholischen Kirche beitragen?
\end{abstract}

\author{
Klaus Große Kracht
}

„Erinnerung kann quälend sein. Namen, Orte, Zeiten, die du verzweifelt versuchst heraufzubeschwören, wollen nicht aus dem Nebel heraustreten. So sehr Du dich bemühst, sie herbeizuzwingen - immer wieder entgleiten dir die Fetzen.“1 Mit diesen Worten beginnt Matthias Katsch sein Buch „Damit es aufhört“. Der Autor, der wie kaum ein zweiter das Thema sexueller Missbrauch in der Kirche aus Sicht der Betroffenen in die Öffentlichkeit gebracht hat, berichtet vom Missbrauch am Berliner Canisiuskolleg, aber auch „vom befreienden Kampf der Opfer sexueller Gewalt in der Kirche“ - so der Untertitel.

In der Tat, wer die Aktenlage in den kirchlichen Archiven kennt, weiß, dass am Anfang einer Fallaufnahme häufig die Meldung durch eine betroffene Person steht, die zwar zum Teil sehr genaue Erinnerungen an ihre Missbrauchserfahrungen hat, zum anderen aber nur über sehr rudimentäres Wissen hinsichtlich des Täters verfügt: War es der „Herr Pastor“, der sie oder ihn aufgefordert hatte, nach der Beichte noch zu bleiben, oder doch der „Kaplan“, war er damals zehn Jahre oder doch schon zwölf Jahre alt? An den Namen erinnert sie sich nur noch flüchtig - aber wie sich die Hände des Täters angefühlt haben, das weiß sie noch sehr genau. So oder ähnlich stellt sich die fragmentarische Erinnerungslage vieler Betroffener häufig dar.

Diese Situation wiederholt sich in den Interviews und Gesprächen mit Betroffenen sexualisierter Gewalt. Nicht selten erzählen sie, dass sie das Erlebte über Jahrzehnte verdrängt und zugleich starke psychische Probleme davongetragen hätten, deren Ursache erst sehr viel später - häufig im Zuge einer Psychotherapie - erkannt wurde. Wenn die betroffenen Kinder im Verlauf der Missbrauchshandlungen nicht anders konnten, als zum Mittel der Dissoziation zu greifen, also ihren Wahrnehmungsapparat vom realen Geschehen abzukoppeln, ist es umso schwieriger, später konkrete Erinnerungen an die Tat abzurufen. So gibt es Betroffene, die heute nicht mehr genau angegeben

1 Matthias Katsch, Damit es aufhört. Vom befreienden Kampf der Opfer sexueller Gewalt in der Kirche. Berlin 2020, 7. 
können, wie weit der sexuelle Missbrauch während ihrer Kindheit ging - die Glaubhaftigkeit ihrer Aussagen ist dadurch aber noch nicht infrage gestellt.

Angesichts dieser Situation ist es eine wichtige Voraussetzung für eine gelingende Aufarbeitung, wenn den Betroffenen genau jene Wissenselemente zur Verfügung gestellt werden, derer sie bedürfen, um ihr Erlebnis in eine für sie stimmige Erzählung einbetten zu können. Viele Betroffene wollen wissen, was aus dem Täter geworden ist. Wurde er jemals für seine Taten zur Verantwortung gezogen oder wurde er einfach nur versetzt und missbrauchte weitere Kinder? Wer wusste von seinen Taten und warum haben die Vorgesetzten ihn nicht gestoppt? Antworten auf diese Fragen sind wichtig, damit das beständige Bohren in den eigenen Erinnerungen aufhört und das Geschehene in einen breiten Wissenskontext eingeordnet werden kann. Bleibt die betroffene Person mit ihren fragmentarischen Erinnerungen allein, läuft das beständige Grübeln Gefahr, sich in einer zermarternden Dauerschleife festzufahren. Im schlimmsten Fall kommt es zu Selbstvorwürfen, warum man das alles so lange hat mit sich machen lassen und wertet sich dadurch selbst massiv ab. Erst das Wissen darum, dass es weitere Opfer gibt, dass andere weggeschaut haben, bewusst nicht eingeschritten sind, dass der Täter strukturelle Ressourcen des Systems, in dem er sich bewegte, genutzt hat, um sich einen Machtvorteil gegenüber seinen Opfern zu verschaffen - all das und vieles mehr an Informationen kann den Betroffenen die Möglichkeit geben, aus dem Zwang der unverschuldeten Selbstbezüglichkeit auszusteigen und die ebenso soziale wie kognitive Isolation ihrer Erinnerungen, die für den Täterschutz so wichtig waren und sind, zu überwinden.

Und genau deshalb ist historische Aufklärung so wichtig. Historikerinnen und Historiker haben es gelernt, mit fragmentarischem Wissen umzugehen, ein Bild der Vergangenheit zusammenzusetzen, auch wenn dieses Bild Lücken aufweist. Sie wissen, wie man aus Akten verlässliches Wissen herausfiltern kann, aber auch, dass nicht alles, was in den Akten steht, die Realität verlässlich und umfänglich widerspiegelt. Sie haben Verfahren der Interpretation und der Quellenkritik entwickelt und eingeübt, die es erlauben, bei der Aktenüberlieferung zwischen den Zeilen zu lesen und aus dem Notierten Informationen herauszuholen, die der Schreiber nicht intendiert hat. Und sie wissen vor allem eins: Akten dokumentieren nicht nur, häufig sind sie zugleich das Produkt vermachteter Kommunikationsstrukturen. Wenn beispielsweise eine Aktennotiz festhält, dass die Familie eines Betroffenen nach dem Besuch des Weihbischofs, der eine Entfernung des Täterpriesters aus der Gemeinde zugesichert habe, keine weiteren Schritte mehr unternehmen und auch die Staatsanwaltschaft nicht einschalten wolle, so heißt das noch lange nicht, dass dies der authentische Wille der besagten Familie war, sondern möglicherweise 
nur das Resultat geschickter pastoraler Kommunikation und Opferüberwältigung durch die Täterinstitution. Auch die gegenwärtigen Aufarbeitungsbemühungen durch kirchliche Akteure sind von dieser gezielten Führung und Vereinnahmung der Betroffenen - man denke nur an die Instrumentalisierung des Betroffenenbeirats durch den Kölner Erzbischof Woelki Ende 2020 - nicht immer frei. ${ }^{2}$

Die Forschung von Psychologinnen und Psychologen, Psychiaterinnen und Psychiatern, Pädagoginnen und Pädagogen ist wichtig, um das Phänomen und die Folgen des sexuellen Missbrauchs von Minderjährigen besser zu verstehen und um sinnvolle präventive Maßnahmen ergreifen zu können. Die Arbeit von Juristinnen und Juristen ist wichtig, um Täter und Vertuscher dingfest zu machen und die Verantwortung für das Geschehen konkreten Individuen zurechnen zu können. Forschenden mit soziologischen, sozialanthropologischen und geschichtswissenschaftlichen Kompetenzen kommt hingegen die Aufgabe zu, die sozialen Systeme und Interaktionsprozesse, in denen sich der Missbrauch vollzogen hat, auf dessen Ermöglichungsstrukturen hin zu untersuchen. Und diese haben vor allem mit Macht und Mentalitäten zu tun. Beides bedingt sich gegenseitig, denn sexueller Missbrauch ist immer auch Machtmissbrauch, der nicht selten erst durch mentale Strukturen der Autoritäts- und Hierarchieakzeptanz ermöglicht wird.

Während die Sozialwissenschaften vor allem systematisch und generalisierend arbeiten, geht die geschichtswissenschaftliche Forschung eher idiographisch-beschreibend vor, das heißt, sie rekonstruiert individuelle Prozessverläufe. ${ }^{3}$ Von daher liegt es nahe, dass die historische Erforschung des sexuellen Missbrauchs Minderjähriger durch katholische Kleriker bei konkreten Fallanalysen ansetzt und diese möglichst genau zu beschreiben versucht. Das Ergebnis auf dieser ersten, untersten Ebene historischer Untersuchung ist daher zumeist die narrative Sequenzierung anhand eines chronologischen Ereignisablaufs. Die aus den Quellen erhobenen Informationen werden zu einer Darstellung eines stimmig nachvollziehbaren Entwicklungsprozesses zusammengefügt, die im Hinblick auf ihre empirische Gültigkeit

2 Matthias Drobinski, Katholische Katastrophe, in: Süddeutsche Zeitung vom 2.11.2020: „Den Betroffenenbeirat des Erzbistums über den Tisch zu ziehen und ihn ein Gutachten kritisieren zu lassen, das er selbst nicht kennt, war eine infame Aktion." Zu den Hintergründen siehe Daniel Deckers/Thomas Jansen, Missbrauchte Betroffene. Um ein Gutachten über sexuellen Missbrauch nicht veröffentlichen zu müssen, bedient sich der Erzbischof von Köln der Opfer sexuellen Missbrauchs, in: Frankfurter Allgemeine Zeitung vom 14.11.2020.

3 Zur Unterscheidung von „idiographisch“ vs. „nomothetisch“ seit Wilhelm Windelband siehe Klaus Grotsch, Nomothetisch/idiographisch, in: Joachim Ritter/Karlfried Gründer (Hrsg.), Historisches Wörterbuch der Philosophie, Bd. 6. Basel 1984, Sp. 896f. 
dem Anspruch genügen sollte, dass keine Quellenfunde von Gewicht der „narrativen Logik“ des Dargestellten widersprechen. Sollte dies der Fall sein, müsste die narrative Sequenzierung entsprechend geändert werden. ${ }^{4}$ Aus solchen Einzelfallbeschreibungen bzw. Teilnarrationen lassen sich dann auf höherer Ebene neue historische Analysen und Synthesen erzeugen, die sich nicht mehr auf den Einzelfall beziehen, sondern Aussagen über Zusammenhänge, eine Organisation, eine Gesellschaft oder andere kollektive Größen treffen. Geht es aber weiterhin um den Aufweis historischer Entwicklungsprozesse, bleibt die Darstellung notwendigerweise narrativ, denn nur die Narration kann zeitliche Verläufe in ihrer Kontinuität darstellen.

Die Narration ist aber nicht nur das adäquate Darstellungsmittel idiographisch-historischer Forschung, ihr methodisches Potenzial liegt noch auf einer weiteren Ebene. Denn die historische Erzählung ermöglicht es besser als andere Darstellungsformen, scheinbar Widersprüchliches, Sperriges und Fragmentarisches zu einem sinnvollen Ganzen zusammenzubinden. Da die Erzählung nicht nach dem Muster logischer Argumentation aufgebaut ist, sondern nach demjenigen der historischen Erfahrung, können auch widersprüchliche Erkenntnisse, sofern sie sich auf unterschiedliche Zeitpunkte beziehen, miteinander zu einer kohärenten Darstellung verknüpft werden. Die Erzählung lebt geradezu davon, dass sie sich nicht auf eine These reduzieren lässt, sondern Raum für Widersprüchliches, Kontingentes und Uneindeutiges lässt. Der französische Philosoph Paul Ricœur nennt die historische Erzählung daher treffend eine „Synthesis des Heterogenen“ - etwas, das die systematischen Wissenschaften nur durch ein Ausblenden des methodisch Nichtpassenden erzeugen können. ${ }^{5}$

Die historische Erzählung lässt damit, sofern sie sich dem Zwang zur Vereindeutigung entzieht, Räume der Interpretation und der nachträglichen Interpolation von Erinnerungen der Rezipienten zu. Sie bietet einen Rahmen der zeitlichen Orientierung, in den die Lesenden ihre eigenen Erinnerungsbilder und -spuren einordnen können. Im Hinblick auf die Betroffenen sexuellen Missbrauchs, welche ihre Wissenslücken als quälend empfinden, mag dies

4 Siehe Arthur C. Danto, Analytische Philosophie der Geschichte. Frankfurt a. M. 1974; Frank R. Ankersmit, Narrative Logic. A Semantic Analysis of the Historian's Language. Den Haag 1983. Zur Einführung in die Theorie historischen Erzählens siehe Achim Saupe/Felix Wiedemann, Narration und Narratologie. Erzähltheorien in der Geschichtswissenschaft (Version 1.o), in: Docupedia-Zeitgeschichte, 28.1.2015, DOI: 10.14765/zzf.dok.2.58o.v1, einzusehen unter http://docupedia.de/zg/saupe_wiedemann_narration_vi_de_2015, zuletzt aufgerufen am 17.5.2021.

5 Paul Ricœur, Zeit und Erzählung, Band 1. Zeit und historische Erzählung. München 1988, 104-113. 
nicht wenig sein. Diese Anheftungsmöglichkeit subjektiver Eindrücke besteht umso mehr, je mehr konkrete Angaben zum zeitlichen Geschehen, zu den Umständen und zu den beteiligten Akteuren mitgeteilt werden. Die Geschichte der Täter und Vertuscher darf nicht im luftleeren Raum verbleiben, wenn sie den Betroffenen einen Dienst erweisen soll.

Welche Anforderungen eine solche, fallbezogene narrative Rekonstruktion sexuellen Missbrauchs Minderjähriger im Bereich der katholischen Kirche erfüllen sollte, um den Kriterien geschichtswissenschaftlicher Standards zu genügen und zugleich eine Anschlussmöglichkeit für weitere, auf einer abstrakteren Stufe angesiedelte historische Synthesen zu ermöglichen, soll im Folgenden anhand von drei Überlegungen zur Diskussion gestellt werden.

\section{Dichte Beschreibung}

Der Ansatz der „dichten Beschreibung“ ist bekannt aus der ethnologischen Forschung. Der Kulturanthropologe Clifford Geertz bezeichnet damit das Verfahren einer bedeutungsorientierten Beschreibung sozialer Prozesse, das nicht nur Verläufe chronologisch verzeichnet, sondern sie als eine "geschichtete Hierarchie bedeutungsvoller Strukturen" versteht. ${ }^{6}$ Bezogen auf den sexuellen Missbrauch innerhalb der katholischen Kirche heißt dies, dass es im Sinne geschichtswissenschaftlicher Aufklärung mit dem bloßen Aufweis von Straftatbeständen aufseiten der Täter und Vertuscher nicht getan ist, sondern die handlungsleitenden Motive der beteiligten Akteure mit erfasst werden müssen. Hierbei geht es nicht allein um die zumeist nur angenommene und vorausgesetzte sexuelle Devianz des Täters, sei diese nun pädophiler, narzisstischer oder schlicht regressiver Natur, sondern auch darum, die Bedeutungskontexte seiner Tat zu erschließen. Wie rechtfertigt er diese vor sich selbst und anderen, vor allem gegenüber dem Opfer? Mit Selbstmitleid, pastoraler Fürsorge oder gar der „Liebe Gottes“? Aufseiten der Vertuscher ist danach zu fragen, ob über den Wunsch des Schutzes der Organisation hinaus spezifische ideologische Momente des Kirchenbildes vorliegen, welche dem Priester oder gar dem Sakrament mehr Schutzrechte einräumen als den betroffenen Gläubigen. Und schließlich ist auch danach zu fragen, inwieweit die kirchliche Seelsorge an

6 Clifford Geertz, Dichte Beschreibung. Bemerkungen zu einer deutenden Theorie von Kultur, in: ders., Dichte Beschreibung. Beiträge zum Verstehen kultureller Systeme. Frankfurt a. M. 1987, 7-43, hier 11. Den Terminus „dichte Beschreibung“ übernimmt er von dem britischen Philosophen Gilbert Ryle (ebd., 10). 
Minderjährigen über eine lange Zeit eine spezifisch katholische Vulnerabilität erzeugt hat, welche der Täter schließlich ausnutzen konnte.

Aus der Perspektive des Betroffenen schreibt Martin Schmitz dazu:

\begin{abstract}
„Immer wieder habe ich mir die Frage gestellt, was den Missbrauch [in] meinem eigenen Leben möglich gemacht hat und immer wieder komme ich auf die eine Antwort, ich war katholisch! Als Kind bin ich mit den Geschichten der Bibel aufgewachsen, mit Adam und Eva, die die Erbsünde in die Welt gebracht haben. Verstanden habe ich das nicht, habe aber erkannt, dass ich vor Gott ein Sünder bin, ein schlechter Mensch, warum auch immer. An anderer Stelle in der Bibel soll Abraham seinen Sohn opfern, um Gott zu gefallen. Da wird es doch bestimmt einen tieferen Sinn haben, wenn mein Vater mich im Alkoholrausch windelweich prügelt oder wenn ein Mann Gottes, für den ich irgendwie ja auch ein Sohn bin, mich missbraucht und vergewaltigt. Und schließlich opfert Gott sogar seinen eigenen, geliebten Sohn am Kreuz für die Sünden der Menschen. Ein allmächtiger Gott, der das bestimmt auch anders hätte lösen können, lässt seinen eigenen Sohn hinrichten. Was durfte ich mich als ,Sohn' denn da beklagen. Ich hatte es auszuhalten, war ich doch der Schuldige allein dadurch, dass es mich gab."
\end{abstract}

Dieser von Schmitz hier eindrücklich beschriebene katholische „Sündenpessimismus" (Ernst Troeltsch) wurde von Eltern, Religionslehrern und Dorfpfarrern an ihre „Schützlinge“ weitergegeben, von Generation zu Generation. Wer jeden Sonntag in der Kirche zu beten gelernt hat, „Herr, ich bin nicht würdig, dass Du eingehst unter mein Dach“, wird kaum das Selbstbewusstsein entwickelt haben, selbst den bizarrsten Aufforderungen des „Hochwürden“ ein deutliches "Nein“ entgegenzusetzen. ${ }^{8}$ Prävention hat daher viel fundamentaler anzusetzen als bei der Frage, wie viele Erwachsene mit wie vielen Kindern in einem Raum zusammen sein dürfen. Und nicht zuletzt der katholischen Theologie und insbesondere dem Lehramt käme die Aufgabe zu, ihren eigenen Traditionsbestand daraufhin zu untersuchen, inwiefern dieser dazu beigetragen hat, dass Kinder in ihrem Selbstbewusstsein geschädigt wurden. Der "spirituelle Missbrauch“ ging dem sexuellen häufig voraus. ${ }^{9}$

Die „Hierarchie bedeutungsvoller Strukturen“, von der Geertz spricht, lässt sich in den überlieferten Dokumenten allerdings nicht einfach auffinden, sie muss vielmehr durch hermeneutische Verfahren mühsam rekonstruiert werden,

7 Martin Schmitz, Weil ich katholisch war! Über das Versagen der Kirche - Bericht eines Betroffenen, in: Thema Jugend. Zeitschrift für Jugendschutz und Erziehung 3, 2020, 6ff., hier 7 .

8 Der in Anlehnung an Mt 8,8 formulierte Vers wird in jeder Eucharistiefeier vor Empfang der heiligen Kommunion von den Gottesdiensteilnehmerinnen und -teilnehmern gebetet.

9 Doris Wagner, Spiritueller Missbrauch in der katholischen Kirche. Freiburg 2019. 
häufig aus einer äußerst fragmentarischen Überlieferung: „Ethnographie betreiben gleicht dem Versuch, ein Manuskript zu lesen (im Sinne von, eine Lesart entwickeln'), das fremdartig, verblasst, unvollständig, voll von Widersprüchen, fragwürdigen Verbesserungen und tendenziösen Kommentaren ist [...]."10 Für die konkrete empirische Forschung bedeutet dies, dass zum Teil sehr genau auch das Unvollständige und die „fragwürdigen Verbesserungen“, von denen Geertz hier spricht, zur Kenntnis genommen werden müssen. Häufig sind es nur scheinbare Details - ein Datum, ein Unterschriftenkürzel -, welche wichtige Informationen enthalten, etwa im Hinblick auf die Zirkulation von Wissensbeständen über Straftaten innerhalb der kirchlichen Personalabteilung oder eines Generalvikariats. Insbesondere die äußere sprachliche Gestalt eines mitgeteilten Sachverhaltes kann dabei zum Teil sehr weitreichende Rückschlüsse auf die dahinterliegenden Interpretationsrahmen ermöglichen: Wird ein Missbrauchstäter etwa als ein Geistlicher bezeichnet, der „einmal kurz vor einer Anzeige stand, weil er unvorsichtigerweise etwas mit Jungs angefangen hat", so zeigt sich die Bagatellisierung der Tat schon in der Ausdrucksweise. Wenn demgegenüber die gelebte Homosexualität von Priestern - unabhängig vom Alter ihrer Partner - jahrhundertelang überhaupt als il pessimo (das Allerschlimmste) bezeichnet wurde, tritt die innerkirchliche Bedeutungshierarchie untermittelbar zutage. ${ }^{11}$

Solche Spuren der Wertung, der Bedeutung und der semantischen Hierarchisierung sind kein kulturhistorisches Beiwerk, auf das eine Aufarbeitung, die diesen Namen verdient, verzichten kann, sondern Indizien eines unsichtbaren Dispositivs, welches die unzureichende Umgangsweise mit sexuellem Missbrauch im Bereich der katholischen Kirche über Jahrhunderte aufrechterhalten und stabilisiert hat. Der italienische Historiker Carlo Ginzburg - der Vorreiter einer am Einzelfall orientierten microstoria - spricht daher auch vom "Indizienparadigma“, das seinen Forschungen zugrunde liege: „Charakteristisch für dieses Wissen ist die Fähigkeit, in scheinbar nebensächlichen empirischen Daten eine komplexe Realität aufzuzeigen $[\ldots]^{\prime}{ }^{“}{ }^{2}$

\footnotetext{
10 Geertz, Dichte Beschreibung, 15.

11 Siehe dazu Hubert Wolf, Zölibat. 16 Thesen. München 2019, $128 \mathrm{f}$.

12 Carlo Ginzburg, Spurensicherung. Der Jäger entziffert die Fährte, Sherlock Holmes nimmt die Lupe, Freud liest Morelli - die Wissenschaft auf der Suche nach sich selbst, in: ders., Spurensicherung. Die Wissenschaft auf der Suche nach sich selbst. Berlin 1995, 7-44, hier 16 und 18.
} 


\section{Kontexte der Bedeutung}

Einzelfälle an sich sind aber noch nicht aussagekräftig. Das Gebot „dichter Beschreibung", die "geschichtete Hierarchie bedeutungsvoller Strukturen" herauszuarbeiten, kann nur gelingen, wenn der Einzelfall in größere soziale und semantische Kontexte eingebettet wird. Sprache und Bedeutung entwickeln sich immer erst in sozialer Interaktion und das gilt auch für die soziale Bewertung des sexuellen Missbrauchs von Kindern. Dieser war seit der Christianisierung Europas grundsätzlich geächtet, auch wenn die Vorstellung davon, was Kindheit als schützenswertes Gut bedeutete, einem erheblichen historischen Wandel unterlag. Gewandelt haben sich zudem die gesellschaftlichen Bilder der Täter und Opfer des sexuellen Missbrauchs. Lange Zeit war die Wahrnehmung der Täter auf die Gestalt des ortsfremden Unholds konzentriert, der als unsublimierter "Triebtäter" im Wald auf der Suche nach kleinen Mädchen unterwegs war. Erst seit den 198oer Jahren erkannte die Öffentlichkeit, in welchem Ausmaß auch die respektablen Väter dieser Mädchen selbst Täter waren. Die Erkenntnis, dass auch minderjährige Jungen in erheblichem Maße Opfer sexualisierter Gewalt geworden sind, hat sich erst in den letzten Jahrzehnten durchgesetzt. ${ }^{13}$

Selbst die wissenschaftliche Forschung im Bereich der Psychiatrie, Pädagogik und Sexualwissenschaft hat lange Zeit gebraucht, um einen angemessenen Blick nicht nur auf die Täter, sondern vor allem auf die Opfer zu artikulieren. Der Expertendiskurs war keineswegs frei von Anmaßungen und Verharmlosungen im Hinblick auf die Beeinträchtigungen und Folgeschäden, welche die an Minderjährigen verübte sexualisierte Gewalt nach sich ziehen konnte. Hinzu kommen personelle und diskursive Überschneidungen mit dem pädosexuellen Milieu, das sich in den in den 1970er und 1980er Jahren im Zuge angeblicher gesellschaftlicher Emanzipationsbestrebungen auch politisch Gehör verschaffen konnte. Diese Sachverhalte sind inzwischen gut aufgearbeitet. ${ }^{14}$

13 Dirk Bange, Geschichte, in: ders./Wilhelm Körner (Hrsg.), Handwörterbuch Sexueller Missbrauch. Göttingen 2002, 135-142; Arno Görgen/Maria Griemmert/Sebastian Kessler, Sexueller Missbrauch und Kinderschutz - Perspektiven im Wandel, in: Jörg M. Fegert u.a. (Hrsg.), Sexueller Missbrauch von Kindern und Jugendlichen. Ein Handbuch zur Prävention und Intervention für Fachkräfte im medizinischen, psychotherapeutischen und pädagogischen Bereich. Berlin 2014, 28-40.

14 Meike Sophia Baader, Blinde Flecken der Disziplin und ihrer Geschichte. Die Involviertheit der Wissenschaft in pädosexuelle Diskurspositionen der 196oer bis 199oer Jahre, in: Karin Amos/Markus Reiger-Ladich/Anne Rohstock (Hrsg.), Erinnern, Umschreiben, Vergessen. Die Stiftung des disziplinären Gedächtnisses als soziale Praxis. Weilerswist 2019, 
Aber auch das Strafrecht und mehr noch die Rechtsprechung bleiben kritisch zu befragen. Sexuelle Handlungen an Kindern unter 14 Jahren waren seit dem Kaiserreich strikt und unmissverständlich verboten, in Bezug auf den Schutz von Jugendlichen über diese Altersgrenze hinaus war und ist die Lage nicht ganz so eindeutig. ${ }^{15}$ Hinzu kommt, dass das Sexualstrafrecht insgesamt von vielen mehr oder weniger aufgeklärten Zeitgenossen in seiner moralischen Verpflichtung nicht wirklich anerkannt wurde, was mit Blick auf die lange Geltung des berüchtigten $§ 175$, der bis 1969 homosexuelle Kontakte zwischen Männern und bis 1994 mit Jugendlichen unter 18 Jahren mit Strafe bedrohte, durchaus verständlich ist. ${ }^{16}$ Ein gesetzlicher Rahmen, der sich mit den normativen Einstellungen der Bevölkerung aber nur in Teilen deckt (Homosexualität), richterlichem Ermessen einen relativ großen Spielraum lässt und die Beweislast allein den Klägerinnen und Klägern aufbürdet, kann jedoch nur eingeschränkt die Funktion eines Warn- und Alarmsystems für potenzielle Täter bieten. Das gleiche gilt übrigens auch für das kirchliche Strafrecht, dem die geweihten Täter unterstanden: Auch dieses verlor nach dem Zweiten Vatikanischen Konzil an Bedeutung und kam häufig nur noch als Ultima Ratio zur Anwendung, sofern die „mitbrüderliche Ermahnung“ nicht mehr weiterhalf. ${ }^{17}$

254-276; Franz Walter/Stephan Klecha/Alexander Hensel (Hrsg.), Die Grünen und die Pädosexualität. Eine bundesdeutsche Geschichte. Göttingen 2015 .

15 Siehe etwa die differenzierten und für den Laien nicht leicht nachvollziehbaren Bestimmungen in der aktuellen Fassung von $\S 182$ StGB (sexueller Missbrauch von Jugendlichen) oder auch die Definition in $\S 184 \mathrm{~h}$, nach der "sexuelle Handlungen“ im Sinne des Gesetzes nur solche seien, „die im Hinblick auf das jeweils geschützte Rechtsgut von einiger Erheblichkeit sind“. Zur uneindeutigen Rechtslage im Hinblick auf die über 14-Jährigen insgesamt siehe Harry Willekens, Der rechtliche Umgang mit der Sexualität von Jugendlichen und Kindern. Widersprüchliche Entwicklungen, in: Meike Sophia Baader u.a. (Hrsg.), Tabubruch und Entgrenzung. Kindheit und Sexualität nach 1968. Köln 2017, 123-135.

16 Zwischen 1969 (überarbeitet im Jahr 1973) und 1994 war der Paragraf de facto eine reine Jugendschutzvorschrift, wobei im Hinblick auf Homosexualität das Schutzalter wesentlich höher angesetzt wurde als im Hinblick auf heterosexuelle Kontakte. Zur Geschichte der Reform des Paragrafen 175 StGB siehe Michael Kandora, Homosexualität und Sittengesetz, in: Ulrich Herbert (Hrsg.), Wandlungsprozesse in Westdeutschland. Belastung, Integration, Liberalisierung 1945-1980. Göttingen 2002, 379-401; sowie zur Diskussion um die Sexualstrafrechtreform der 196oer und 1970er Jahre insgesamt Franz Walter, ,In dubio pro libertate'. Sexualstrafrecht im gesellschaftlichen Wandel, in: ders./Klecha/ Hensel (Hrsg.), Die Grünen und die Pädosexualität, 108-135.

17 CIC (1983), can. 1341. Peter Platen, Das kirchliche Strafrecht. Eine (leider?) vernachlässigte Disziplin. Überlegungen zur kirchenrechtlichen Ahndung des sexuellen Missbrauchs Minderjähriger durch Geistliche, in: Kirche \& Recht 16, 2010, 192-208. 
Eng mit diesen unübersichtlichen wissenschaftlichen und rechtlichen Grenzziehungen verknüpft vollzog sich in den 196oer und 197oer Jahren die sogenannte sexuelle Revolution. Grenzverschiebungen sprachlicher, visueller und praktischer Art im Umgang mit Sexualität waren nun nicht nur an der Tagesordnung einer antibürgerlichen jugendlichen Revolte, sondern auch in anderen Teilen der Bevölkerung avancierte das Prinzip der „sexuellen Selbstbestimmung“ zum entscheidenden Kriterium des geschlechtlichen Miteinanders. ${ }^{18}$ Traditionell verfestigte und asymmetrische Beziehungsstrukturen der "heterosexuellen Matrix" (Judith Butler) konnten dadurch nachhaltig durchbrochen werden. Im Hinblick auf Kinder und Minderjährige führte die Fokussierung auf das sexuelle Selbstbestimmungsrecht allerdings auch dazu, die Augen vor der Schutzbedürftigkeit derjenigen zu verschließen, die dieses Recht nicht ausüben können. Angeblich sollten auch kleine Kinder in der Lage sein, selbst zu entscheiden, wann und mit wem sie sexuelle Kontakte erleben wollten. ${ }^{19}$

Die kirchliche Auseinandersetzung mit dem Thema des sexuellen Missbrauchs bewegte sich entlang dieser allgemeinen gesellschaftlichen Wahrnehmungsverläufe und muss entsprechend kontextualisiert werden, wenn man die Motive der Vertuscher verstehen will. Denn zum einen sind auch die Gottesmänner zunächst einmal Kinder ihrer Zeit und über Jahrhunderte deckte sich die kirchliche Morallehre mit den Sittlichkeitsvorstellungen der sie umgebenden Mehrheitsgesellschaft. Je mehr sich die gesellschaftlichen

18 Peter-Paul Bänziger u.a. (Hrsg.), Sexuelle Revolution? Zur Geschichte der Sexualität im deutschsprachigen Raum seit den 196oer Jahren. Bielefeld 2015; Dagmar Herzog, Die Politisierung der Lust. Sexualität in der deutschen Geschichte des zwanzigsten Jahrhunderts. Berlin 2005, 173-222.

19 Selbst ein so sehr an der Beobachtung subtiler Machtverhältnisse interessierter Sozialanalytiker wie Michel Foucault sprach sich 1978 dafür aus, auf die Festlegung von altersgemäßen Schutzfristen grundsätzlich zu verzichten und es stattdessen dem „Kind“ zu überlassen, „selbst zu sagen, ob ihm Gewalt angetan worden ist oder nicht“, zitiert nach Baader, Blinde Flecken, 258. Gegen Foucault sind vor kurzem zudem - bislang allerdings unbewiesene - Anschuldigungen erhoben worden, er habe selbst Minderjährige missbraucht, siehe Georg Blume, Foucaults tunesische Jungen, in: Die Zeit, 7.4.2021. Auch Theodor W. Adorno mokierte sich bereits 1963 über den "Minderjährigenkomplex“, der dazu führe, „auf jeden Spielplatz hinter jedes Kind eine sittlich gereifte Polizistin stellen“ zu wollen, und schwadronierte des Weiteren über eine unterschwellige „Homosexualisierung der Gesellschaft“, durch die das „erotische Ideal infantil werde“, siehe Theodor W. Adorno, Sexualtabus und Recht heute, in: Fritz Bauer (Hrsg.), Sexualität und Verbrechen. Beiträge zur Strafrechtsreform. Frankfurt a. M. 1963, 299-317, hier 309. Zur Diskussion kindlicher Sexualität im Zuge der „sexuellen Revolution“ siehe Jens Eberfeld, Von der Sünde zur Selbstbestimmung. Zum Diskurs ,kindlicher Sexualität' (Bundesrepublik Deutschland 1960-199o), in: Bänziger u.a. (Hrsg.), Sexuelle Revolution, 247-283. 
Normvorstellungen von ihrer kirchlichen Engführung aber emanzipierten, desto spannungsreicher wurde das Verhältnis zwischen kirchlichen und säkularen Lebensentwürfen. Diese Spannung wirkte schließlich auf die Entwicklung der kirchlichen Sexualnorm selbst zurück und führte zu Verhärtungen, welche den Gläubigen in einer gewandelten gesellschaftlichen Umwelt kaum noch sinnvolle Orientierung zu geben vermochten. ${ }^{20}$ So reagierte das Lehramt im Jahr der Studentenunruhen 1968 mit einer demonstrativen Bekräftigung ihrer traditionellen Sexualmoral, indem Papst Paul VI. in seiner Enzyklika „Humanae vitae“ die künstliche Empfängnisverhütung strikt verbot und damit die legitime Ausübung von Sexualität für die Gläubigen auf den Bereich des heterosexuellen, unverhüteten, ehelich-monogamen Verkehrs beschränkte. ${ }^{21}$ 1976 zog der Vatikan noch einmal nach und ließ durch eine Erklärung der Glaubenskongregation Sex vor und außerhalb der Ehe, praktizierte Homosexualität und Masturbation erneut als „schwere Sünde“ strikt verurteilen. ${ }^{22}$

Wer die Grenzen dermaßen eng zog, entfernte sich aber immer mehr von einer Gesellschaft, welche den Raum akzeptierter Sexualpraktiken im Gegensatz zur Kirche zunehmend ausdehnte und dafür mit dem Rekurs auf das Selbstbestimmungsrecht des Menschen gute Gründe anführen konnte. Die verklausulierte Sprache von der "prägenden Kraft der Keuschheit" und einer „Begierde, die in der Erbsünde gründet“, verstanden hingegen immer weniger Menschen, ${ }^{23}$ zumal viele Katholikinnen und Katholiken schon vor „Humanae vitae" der traditionellen katholischen Morallehre den Rücken gekehrt hatten. ${ }^{24}$

Man wird davon ausgehen können, dass auch unter den Priestern die Zahl derer wuchs, welche die rigiden Sexualnormen, die sie im Theologiestudium und Priesterseminar vermittelt bekommen hatten, als lebensfremd empfanden. In dieser Situation wurden sie von der kirchlichen Führungselite alleingelassen: Während Papst und Bischöfe das zölibatäre Priesterbild kultisch

$20 \quad$ Anders übrigens die evangelische Kirche, die auf die gewandelten Moralvorstellungen 1971 mit einer behutsamen und wesentlich differenzierter argumentierenden Denkschrift zu Fragen zur Sexualethik reagierte, siehe Herzog, Politisierung der Lust, 185.

21 Siehe Birgit Aschmann/Wilhelm Damberg (Hrsg.), Liebe und tu, was du willst? Die ,Pillenenzyklika' Humanae vitae von 1968 und ihre Folgen. Paderborn 2021.

22 Die Stellung der Kirche zu sexualethischen Fragen, in: Herder-Korrespondenz 30, 1976, $82-87$.

23 Ebd., 87. In einer erläuternden „Stellungnahme des Vorsitzenden der Deutschen Bischofskonferenz" gab selbst dieser zu bedenken, dass die „deduktive Argumentationsweise [...] manchem das Verständnis und den Zugang zum Inhalt der ,Erklärung‘ erschweren“ könnte. „Manche werden in verschiedenen Punkten eine differenziertere und ausführlichere Behandlung vermissen“ (ebd., 88).

24 Lukas Rölli-Alkemper, Familie im Wiederaufbau. Katholizismus und bürgerliches Familienideal in der Bundesrepublik Deutschland, 1945-1965. Paderborn 200o, 194-225. 
überhöhten, ${ }^{25}$ versäumten sie es, den Klerikern praktikable Maßstäbe der ethischen Differenzierung für den Alltag an die Hand zu geben: Wenn Sexualität für sie eh tabu war und jedwede weitere normative Unterscheidung - etwa zwischen gelebter Homosexualität auf der einen Seite und dem sexuellen Missbrauch von Kindern auf der anderen Seite ${ }^{26}$ - nicht mehr weiter ins Gewicht fiel, bewegte man sich kognitiv bereits in der Gefahrenzone. Beides war aus der Sicht der Kirche gleichermaßen eine schwere Sünde, und wer bereits in ihrem Zustand lebte, hatte vor Gott und seinem Stellvertreter nicht mehr viel zu verlieren.

\section{Zeitschichten der Bedeutung}

Die "geschichtete Hierarchie bedeutungsvoller Strukturen" baut sich aber nicht nur im Kontakt mit der sozialen Umwelt auf. Neben der horizontalen Ausbreitung in den gesellschaftlichen Raum hinein verteilt sie sich vertikal über unterschiedliche „Zeitschichten“. ${ }^{27}$ Seit der grundlegenden Arbeit des französischen Sozialhistorikers Fernand Braudel ist es üblich geworden, in der Geschichtswissenschaft zumindest drei solcher Zeitschichten zu unterscheiden: das Ereignis, die mittlere Dauer und die lange Dauer (longue durée). Auf der Zeitebene des Ereignisses siedelte Braudel historische Prozesse von kurzer zeitlicher Erstreckung wie Schlachten, Herrschaftswechsel etc. an, auf der Ebene der mittleren Dauer demografische Bewegungen, wirtschaftliche Konjunkturzyklen und Strukturen der sozialen Schichtung sowie schließlich im Bereich der langen Dauer lang anhaltende, quasi stabile Strukturformen

25 Noch im Jahr 2010 sprach Erzbischof Joachim Kardinal Meisner anlässlich des Silbernen Priesterjubiläums seines damaligen Weihbischofs Rainer Maria Woelki vom Pfarrer als dem „Engel seiner Gemeinde“: In ihm begegneten die Menschen Gott, einzusehen unter https://www.erzbistum-koeln.de/erzbistum/erzbischof/amtsvorgaenger/joachim_ meisner/predigten_hirtenworten_ansprachen/Predigten/jcm_pr_10o613_woelki-jubil. pdf, zuletzt aufgerufen am 17.5.2021. Zu den Problemen eines überhöhten Priesterbildes siehe mit weiteren Belegen Norbert Lüdecke, Sexueller Missbrauch von Kindern und Jugendlichen durch Priester aus kirchenrechtlicher Sicht, in: Münchener Theologische Zeitschrift 62(1), 2011, 33-6o.

26 Die Instruktion „Crimen sollicitationis“ (1922/1962), die zumindest bis zur Promulgation des überarbeiteten Codex Iuris Canonici im Jahr 1983 in Kraft blieb, bezeichnete den sexuellen Verkehr zwischen einem Priester und einem Mann als crimen pessimum (das schlimmste Verbrechen), dem der sexuelle Missbrauch Minderjähriger und die Sodomie gleichgestellt wurden, einzusehen unter http://www.vatican.va/resources/resources_ crimen-sollicitationis-1962_en.html, § 71-74, zuletzt aufgerufen am 17.5.2021.

27 Reinhart Koselleck, Zeitschichten. Studien zur Historik. Frankfurt a. M. 200o, 19-26. 
des Wirtschaftens und der Verkehrswege bis hin zu geografischen und klimatischen Begebenheiten. ${ }^{28}$

Dieses Modell lässt sich auch auf die geschichtswissenschaftliche Untersuchung von Fällen sexuellen Missbrauchs im Bereich der katholischen Kirche übertragen, wenn auch sicherlich nicht ganz so schematisch: Im Hinblick auf das „Ereignis" stehen dann die eigentlichen Missbrauchstaten im Vordergrund, aber auch die spezifischen Formen der Anbahnung und der Verdunkelung. Das Wissen hierum lässt sich nur teilweise aus der Aktenüberlieferung entnehmen. Gerade in dieser Hinsicht sind die Forschenden auf die Berichte der Betroffenen angewiesen. Dass es im Gespräch mit diesen aber nicht einfach damit getan ist, die Prinzipien der oral history oder der qualitativen Sozialforschung zu beachten, versteht sich von selbst. Der Respekt vor den Betroffenen bringt es vielmehr mit sich, ihren Erzählungen mit allen Brüchen und Umwegen zu folgen, auch um den Preis, sich vom vorgefertigten InterviewLeitfaden zu lösen. Hinzu kommt, dass die interviewte Person das angetane Leid möglicherweise dissoziiert haben könnte, sodass sich das konkrete Tatgeschehen nicht mehr im Einzelnen rekonstruieren lässt. Sollte das der Fall sein, wird man die dadurch entstandene „Lücke“ im Geschehensablauf nicht anders als hinnehmen können; sie sollte aber weder Anlass für Spekulationen geben noch dazu, die Glaubwürdigkeit insgesamt in Abrede zu stellen. ${ }^{29}$

Auf der Ebene der "mittleren Dauer" geraten die Biografien von Tätern und Opfern in den Blick. Denn die Folgen sexuellen Missbrauchs geben sich häufig erst viele Jahre später zu erkennen. Nicht selten treten sie erst dann ins Bewusstsein, wenn es darum geht, die Gründe zu erkunden, warum das eigene Leben weniger gelungen verlaufen ist als das naher Mitmenschen, warum Depressionen, Bindungsängste, mangelndes Selbstbewusstsein und vieles mehr bis hin zu Suizidversuchen den eigenen Lebensentwurf durchkreuzt haben. Aber auch im Hinblick auf den Täter ist die Betrachtung seiner gesamten Biografie unabdingbar: Hat er selbst sexuelle Gewalt erfahren? Gab es Anzeichen für das von ihm ausgehende Gefahrenpotenzial? Wie verlief sein

28 Fernand Braudel, Das Mittelmeer und die mediterrane Welt in der Epoche Philipps II., 3 Bde. [1949]. Frankfurt a. M. 199o; ders., Geschichte und Sozialwissenschaften. Die longue durée, in: Marc Bloch/Fernand Braudel/Lucien Febvre, Schrift und Materie der Geschichte. Vorschläge zu einer systematischen Aneignung historischer Prozesse, hrsg. von Claudia Honegger. Frankfurt a. M. 1977, 47-85.

29 Glaubwürdigkeitsprüfungen sind selbstverständlich wichtig, sollten aber der forensischen Psychiatrie überlassen bleiben. Zur kulturwissenschaftlichen Interviewsituation siehe Natalie Powroznik, Ich will mich nicht mehr verstecken. Die Aufarbeitung des Missbrauchs an Minderjährigen im Bistum Münster, in: Thema Jugend. Zeitschrift für Jugendschutz und Erziehung 3, 2020, $3 \mathrm{ff}$. 
Leben nach dem Missbrauch? War er ein Mehrfachtäter, der trotz besseren Wissens aufseiten seiner Personalvorgesetzten nicht gestoppt wurde?

In geschichtswissenschaftlicher Hinsicht von besonderer Bedeutung ist schließlich die Betrachtung der "langen Dauer“. Dies betrifft insbesondere die Geschichte der Kirche als religiöse Institution. Damit ist sowohl ihre rechtlich-formale Organisation gemeint als auch das institutionelle Normengefüge, das durch religiöse Sozialisation, Ausbildung und kleinräumliche Sozialbeziehungen an die Gläubigen weitergegeben wird und sich damit wie ein unsichtbares Netz über Opfer, Täter und Mitwissende legt und diese aneinanderbindet. ${ }^{30}$ Diese „lange Dauer" entspricht übrigens auch dem Selbstbild der Kirche, die sich als eine übergeschichtliche Institution, als Corpus mysticum, versteht. Daher unterliegt sie in dogmatischen Fragen auch nicht dem historischen Wandel, sondern war und ist immer im Besitz der Wahrheit. Die Fiktion dieses „Dispositivs der Dauer“31 führt dazu, dass die Möglichkeit radikaler Kritik und Veränderung im Vorhinein ausgeschlossen und der einmal eingeschlagene Weg trotz nagender Selbstzweifel fortgesetzt wird: Dass der Zölibat nicht abgeschafft wird, Frauen nicht zum Priesteramt zugelassen werden, die Demokratie der Kirche wesensfremd sei - dies alles wird mit Blick auf die Autorität der Tradition als Hort des depositum fidei begründet, über das man sich nicht hinwegsetzen dürfe.

Ausdruck dieser "langen Dauer“ des institutionellen Selbstverständnisses ist nicht zuletzt das Kirchenrecht, das nicht nur ein formales Regelwerk innerkirchlicher Abläufe darstellt, sondern in einem theologisch begründeten Bild der Kirche wurzelt, deren primäre Aufgabe darin gesehen wird, das Seelenheil der Gläubigen zu sichern. Der letzte Eintrag im Codex Iuris Canonici von 1983 macht dies sehr deutlich, wenn hier - an prominenter Stelle - die Sorge um das „Heil der Seelen“ (salus animarum) als das „oberste Gesetz“ der Kirche bezeichnet wird. ${ }^{32}$ Dieses Seelenheil wird nach katholischer Auffassung aber vor allem durch den Empfang der Sakramente sichergestellt, weshalb diese unter einem besonderen kirchenrechtlichen Schutz stehen. Sexuelle Übergriffe auf Minderjährige wurden im Kirchenrecht daher entweder als Verstoß gegen die Priesterweihe und die daraus resultierende Verpflichtung zur Einhaltung des Zölibats gewertet oder aber als Beschmutzung des Bußsakraments, sollte etwa eine sexuelle Erregung (sollicitatio) die Sakramentenspende begleitet

30 Zum normativen Begriff der Institution siehe Konstanze Senge/Kai-Uwe Hellmann (Hrsg.), Einführung in den Neo-Institutionalismus. Organisation und Gesellschaft. Wiesbaden 2006.

$31 \quad$ Rainer Bucher, Kirchenbildung in der Moderne. Eine Untersuchung der Konstitutionsprinzipien der deutschen katholischen Kirche im 20. Jahrhundert. Stuttgart 1998, 43-5o. CIC (1983), can. $175^{2}$. 
haben. Das oberste Rechtsgut, das kirchenrechtlich geschützt werden sollte, war also nicht etwa die körperliche und psychische Unversehrtheit der minderjährigen Person, sondern die Reinheit des Sakramentes. Diese Haltung zeigt sich selbst noch in dem päpstlichen Motu proprio „Sacramentorum sanctitatis tutela", mit welchem der Vatikan 2001 mit durchaus rigorosen kirchenrechtlichen Vorschriften auf den schwelenden Missbrauchsskandal in den eigenen Reihen reagierte. Die titelgebenden Anfangsworte des Schreibens lauten in der deutschen Übersetzung: „Der Schutz der Heiligkeit der Sakramente“. Dieser sowie „die Befolgung des sechsten Gebotes“ erforderten, „dass die Kirche, zum Heil der Seelen, ,das in der Kirche immer das oberste Gesetz sein muss' (CIC, can. 1752)“, einschreite und schwerwiegende Verstöße durch die Glaubenskongregation verfolgen lasse. ${ }^{33}$

Sexualisierte Gewalt gegenüber Kindern ist kirchenrechtlich gesehen also nicht so sehr ein Verstoß gegen deren Grund- und Menschenrechte als vielmehr eine Behinderung der vollen Gnadenentfaltung durch das Sakrament, mithin ein Verbrechen gegen Gott. Im kirchlichen „Dispositiv der Dauer“ sind somit fundamentale Denkweisen sedimentiert, welche sich von der strafrechtlichen Bewertung von Fällen sexualisierter Gewalt im staatlichen Strafrecht erheblich unterscheiden. Auch solche Unterschiede müssen herausgearbeitet werden, um zu verstehen, warum kirchliche Personalverantwortliche im Hinblick auf den sexuellen Missbrauch von Minderjährigen durch Priester so gehandelt haben, wie sie gehandelt haben.

\section{Fazit und Postskriptum}

Die Aufarbeitung des sexuellen Missbrauchs an Minderjährigen durch katholische Priester wird auch nach Abschluss der vielen zurzeit unternommenen Untersuchungen zu einzelnen deutschen Diözesen nicht so schnell zu einem Ende kommen. Die Vorfälle waren zu massiv, die Vertuschungsroutinen zu verbreitet, als dass sie das Kirchenbild der Gläubigen unbeschadet lassen könnten. Wenn ein renommierter Kirchenhistoriker die Erschütterung durch den aktuellen Missbrauchsskandal als "größer“ einschätzt

33 Apostolisches Schreiben Motu proprio "Sacramentorum sanctitatis tutela“ von Papst Johannes Paul II., durch das die Normen bezüglich schwerwiegenderer Straftaten, die der Glaubenskongregation vorbehalten sind, promulgiert werden, einzusehen unter http://www.vatican.va/content/john-paul-ii/de/motu_proprio/documents/hf_jp-ii_ motu-proprio_20020110_sacramentorum-sanctitatis-tutela.html, zuletzt aufgerufen am $17.5 \cdot 2021$. 
als die Erschütterung durch die Reformation, dann sollte das zu denken geben: „Eine Religion, die keine Glaubwürdigkeit hat, ist am Ende.“34

$\mathrm{Ob}$ die Kirche ihre Glaubwürdigkeit wieder erlangen kann, wird nicht unwesentlich davon abhängen, ob sie bereit ist, ihr Selbstbild, ihr Amtsverständnis und ihre Ämterhierarchie sowie ihr Bild von der menschlichen Geschlechtlichkeit einer grundsätzlichen Revision zu unterziehen. Das werden die Kirchenmitglieder - Kleriker wie Laien - auf synodalen oder anderen Wegen unter sich ausmachen müssen. Ein Großteil der vom Missbrauch Betroffenen hat der Kirche eh den Rücken gekehrt. Politik und Gesellschaft kommt die Aufgabe zu, sie mit ihren Geschichten nicht allein zu lassen. Dazu kann die geschichtswissenschaftliche Forschung einen Beitrag leisten.

Eine breite sozial- und kulturgeschichtliche Erforschung des sexuellen Missbrauchs an Minderjährigen innerhalb der katholischen Kirche ist aber auch deshalb wichtig, damit sich die aktuelle Debatte nicht allein auf rein juristische Fragen verengt. Zurzeit wird in den von einzelnen Bistumsleitungen in Auftrag gegebenen Gutachten und den darauffolgenden Presseberichten zu möglicherweise versäumten Amtspflichten zum Teil sehr detailliert die Nachlässigkeit, Fahrlässigkeit und Unterlassung aufseiten von Bischöfen, Generalvikaren und Domkapitularen diskutiert, die Beschreibung konkreter Tatsituationen und Täterbiografien aber vermieden. Begründet wird dies mit der Gefahr der Retraumatisierung von Betroffenen und eines angeblichen Voyeurismus aufseiten der Leserinnen und Leser. Aber genau diese „paternalistische Haltung“35 gegenüber den Betroffenen führt dazu, dass die im katholischen Milieu gepflegten diskursiven Schamgrenzen, die es den Betroffenen schon damals, als sie den Missbrauch erlitten, unmöglich machten, darüber zu sprechen, perpetuiert werden. So kann man über juristische Details in der Bewertung von Amtspflichten und äußerungsrechtlichen Details lange und detachiert diskutieren, wenn Missbrauch nur mehr ein Schattenbegriff ist und die konkreten Verbrechen, die verübt wurden, gar nicht mehr zur Sprache kommen. Die Betroffenen können zum Teil aber sehr wohl über das Tatgeschehen sprechen und wo sie es (noch) nicht können, sollte es ihnen ermöglicht werden. Und man sollte ihnen zuhören, denn häufig sind es erst die Details, die sie berichten nicht unbedingt der Tat selbst, aber der sie begleitenden Umstände: etwa die Dose Fanta, die sich der neunjährige Junge jeweils vor dem Missbrauch aus dem Keller des Pfarrhauses holen durfte -, welche die Last seiner Geschichte erst erahnbar machen. 